\title{
O modelo keynesiano de James Meade
}

Claudia Heller

Departamento de Economia, Faculdade de Ciências e Letras - UNESP

Roselaine Bonfim de Almeida Doutoranda em Economia Aplicada - PPGEA/ESALQ/USP

\section{Palauras-chave}

Teoria Keynesiana;

Investimento e Poupança;

Equilíbrio; Estabilidade;

Expectativas

Classificação JEL B22, B31,

E12

\section{Key-words \\ Keynesian theory; Investment and Saving; Equilibrium; \\ Stability; Expectations \\ JEL Classification B22, B31,}

\section{Resumo}

Este artigo reavalia o "modelo simplificado" da Teoria Geral de Keynes elaborado por James Meade e procura complementar alguns dos argumentos teóricos que Meade deixou apenas indicados. $\mathrm{O}$ artigo também responde às solicitações de Frisch a Meade, quanto à explicação do significado de estabilidade e à explicitação dos pressupostos que garantem suas conclusões. Além disso, apresenta alguns dos cálculos não desenvolvidos por Meade, e, quando necessário, suas devidas correções.

\section{Albstract:}

The paper reassesses the "simplified model" of Keynes's General Theory developed by James Meade and attempts to complement some of the theoretical arguments that Meade only left indicated. It also answers Frisch's questions to Meade with regard to explanation of the mean of stability and elaboration of the assumptions that support his conclusions. The paper also presents some of the calculations undeveloped by Meade and, when necessary, their corrections. 


\section{1_Introdução}

Uma das primeiras tentativas de simplificar a Teoria Geral do Emprego, dos Juros e da Moeda de John Maynard Keynes através de um conjunto de equaçóes matemáticas foi a de James Edward Meade, que apresentou sua interpretação no Simpósio "Mr. Keynes' System", durante a Sexta Conferência Europeia da Sociedade de Econometria, realizada em Oxford, de 25 a 29 de setembro de 1936. Também compareceram a este evento Roy Harrod e John Hicks, cujas contribuiçôes ("Mr. Keynes and traditional theory" e "Mr. Keynes and the 'Classics': a suggested interpretation") foram publicadas na revista Econometrica em janeiro e abril de 1937, respectivamente.

Há indícios de que a versão apresentada por Meade no mencionado Simpósio não é a mesma que foi publicada na Review of Economic Studies, em fevereiro de 1937. O primeiro é uma carta de Ragnar Frisch, editor de Econometrica, enviada a Meade em 08 de outubro de 1936 (reproduzida em YOUNG, 1987, p. 36). O outro é uma carta de Ursula Hicks, editora da Review of Economic Studies, enviada a Meade em 30 de novembro de 1936 (também reproduzida em YOUNG, 1987, p. 37). Frisch solicitava uma revisão que incorporasse o "significado exato de estabilidade" e a descriçấo "dos pressupostos que garantem suas [de Meade] conclusões". Ursula Hicks solicitava a inclusão de notas de rodapé e um apêndice matemático, para que "o problema, o argumento e as conclusóes ficassem cristalinamente claros". Aparentemente Meade discordou das modificaçóes sugeridas por Frisch e, em consequência, embora Econometrica tenha publicado a íntegra dos trabalhos de Hicks e de Harrod, só publicou um resumo do de Meade, incorporado ao texto do relatório da Conferência redigido por Phelps-Brown. As sugestóes de Ursula Hicks devem ter sido consideradas como suficientemente contempladas, já que o artigo "A simplified model of Mr. Keynes's system" foi publicado na Review of Economic Studies (com notas de rodapé e um apêndice matemático). ${ }^{1}$ de forma independente, isto é, sem ter lido o de Hicks nem o de Harrod. Por outro lado, Heller (2007) reconhece que é possível que Meade tenha tido a oportunidade de ler outros trabalhos que procuravam representar a Teoria Geral através de formalizaçóes algébricas, como é o caso dos artigos de David Champernowne ("Unemployment, basic and monetary: the Classical analysis and the Keynesian") e de Brian (continua a direita) 
Reddaway ("The General Theory of Employment, Interest and Money"), publicados em junho de 1936, antes da Conferência de Oxford. O fato é que as informaçōes disponíveis sobre a circulação prévia do texto de Meade são precárias. $\mathrm{O}$ único registro é um cartáo postal enviado por Keynes a Meade, datado de 14 de setembro de 1936 (YOUNG, 1987, p. 34 e 189), indicando que Keynes conhecia o trabalho antes da apresentação em Oxford.
Uma leitura cuidadosa do artigo de Meade revela que há um conceito de estabilidade, assim como supostos que sustentam suas conclusóes, embora não estejam tão explícitos como foi solicitado por Frisch; revela, também, que nem sempre os argumentos teóricos que ligam os supostos às conclusôes estão completos. O presente texto procura corrigir essas falhas. Além disso, embora a versão publicada inclua um apêndice matemático, várias expressóes algébricas utilizadas por Meade ao longo do seu artigo não estão suficientemente desenvolvidas nesse apêndice. $\mathrm{O}$ presente texto procura também preencher as principais lacunas matemáticas deixadas por Meade, explicitando as simplificações necessárias para alcançar os resultados

\footnotetext{
2 O termo "MEK" representa o setor produtor de bens de capital e o termo "AA" representa o mercado de ativos.

${ }^{3} \mathrm{p}_{\mathrm{I}}$ e $\mathrm{p}_{\mathrm{S}}$ representam o preço de demanda e o preço de oferta de bens de capital, respectivamente.
}

do autor, e/ou fazendo as devidas correções. É bom ressaltar que o texto não tem a pretensão de comparar a interpretação de Meade com a dos seus contemporâneos nem de apresentar uma nova visão da história da síntese neoclássica, mas apenas contribuir com uma análise acurada de um dos textos que compóem a etapa inicial dessa história que, curiosamente, embora tenha sido negligenciada por meio século, vem sendo recentemente revista por vários autores.

De modo genérico, os intérpretes de Meade procuram reformular seu sistema de equaçóes e traduzi-lo em diagramas (supostamente compatíveis com o modelo IS-LM). Uma das abordagens é a que foi iniciada com o artigo de Darity e Cottrell (1987), dando origem ao diagrama $A A-M E K$, posteriormente ampliada por Alanez (2001). ${ }^{2}$ Outra abordagem é o diagrama $p_{I}-p_{S}$, proposto por Rappoport (1992) e desenvolvido por Assous (2004). ${ }^{3}$ Os dois tipos de diagramas são inspirados na crítica, inicialmente elaborada por Darity e Cottrell (1987), de que no sistema de Meade os detentores de riqueza não têm alternativas - exceto o investimento produtivo - caso queiram abrir mão da liquidez. Por isso, os autores sugerem distinguir o conceito de taxa de juros do de eficiência marginal do capital 
através da introdução explícita de algum tipo de ativo que não é nem moeda nem estoque de capital produtivo. Em outras palavras, introduzem um mercado financeiro que, no sistema original de Meade, estava apenas implícito.

Todos os intérpretes mencionados acima consideram que o modelo proposto por Meade é superior aos seus congêneres - as referências são Hicks (1937) e Harrod (1937) - principalmente por incluir uma variável que representa o estado de expectativas. Essa é uma das características que Darity e Cottrell (1987) ressaltam ao considerarem que o esquema de Meade tem essência keynesiana e é o que justifica a análise proposta por Alanez (2001), que distingue expectativas exógenas de expectativas estáticas no arcabouço de Meade, para avaliar se os resultados são semelhantes aos da abordagem de Hicks (1937) ou aos propostos por Keynes (1936). Rappoport (1992) também enfatiza o papel das expectativas como o elemento central do modelo de Meade, em contraponto ao modelo IS-LM convencional, e Assous (2004) destaca o papel das expectativas na discussão sobre os efeitos da redução de salários sobre o nível de emprego como critério de distinçâao entre modelos de caráter clássico ou keynesiano.

O estudo desses intérpretes recentes foi feito por Almeida (2008), que ob- serva que, a despeito da variedade de recursos algébricos utilizados por esses autores, suas conclusóes não divergem essencialmente dos resultados que Meade alcançou em seu texto original. Por essa razão o presente texto limita-se à análise do "modelo simplificado" de Meade, e procura fazer algumas das devidas complementaçóes aos argumentos que ele deixou inacabados.

O texto está dividido em duas partes, além desta Introdução e da Conclusão. Na primeira explicitam-se o conceito de equilíbrio e as condições de sua estabilidade, conforme solicitado por Frisch. $\mathrm{Na}$ segunda discutem-se os efeitos de mudanças exógenas na taxa de juros, na oferta de moeda, nos salários e na propensão a poupar sobre o nível de emprego, o que permite atender à segunda solicitação de Frisch, de explicar os pressupostos que garantem as conclusóes de Meade. $\mathrm{O}$ desenvolvimento de algumas das expressóes algébricas de Meade (e as eventuais correções) encontra-se nos Anexos.

\section{2_O conceito de estabilidade}

\section{1_ As condições de equilíbrio}

Meade tem por objetivo construir um modelo simples da Teoria Geral do Em- 
prego, dos Juros e da Moeda de Keynes com a finalidade de ilustrar três aspectos:

i. as condições necessárias para o equilíbrio;

ii. as condições necessárias para a estabilidade do equilíbrio; e

iii. o efeito, sobre o emprego, das mudanças em certas variáveis. (MEADE, 1937, p. 98)

O modelo está baseado em suposiçóes simplificadoras que descrevem uma economia fechada em concorrência perfeita com dois setores, um que produz bens de capital e outro que produz bens de consumo, ambos com a mesma elasticidade de oferta de curto prazo. Em cada setor existem apenas dois fatores de produção, trabalho e capital. O fator trabalho representa o único custo direto e o fator capital dura eternamente, pois não se desgasta nem se deprecia. $\mathrm{O}$ dispêndio monetário total em bens de consumo e de investimento constitui a renda nacional que se reparte na forma de salários e lucros.

Embora seja um modelo de curto prazo, definido como "o período no qual a razão entre a produção de novos bens de capital e o estoque existente de bens de capital é pequena, de modo que as alteraçóes no estoque de bens de capital podem ser negligenciadas" (MEADE, 1937, p. 98), o modelo permite ajustes no volume de produção, na taxa de investimento e/ou no montante gasto e poupado de cada indivíduo. $\mathrm{O}$ ajuste no volume de produção permite que o custo marginal de cada bem se iguale ao seu preço e o ajuste da taxa de investimento permite que a taxa de juros se iguale à eficiência marginal do capital. Alteraçóes no dispêndio ou no montante poupado, por sua vez, podem ser decorrentes de mudanças no nível de renda e/ou da propensão a poupar.

Essas suposiçóes formam a base do conjunto de equaçóes que descrevem o modelo: ${ }^{4}$
${ }^{4}$ Essas equaçóes constam do apêndice matemático fornecido por Meade (1937, p. 105), mas a notação utilizada neste texto é um pouco diferente da notação do autor. No lugar dos subscritos " $\mathrm{x}$ " e " $y$ " usamos os subscritos "I" e "C" para designar os setores produtores de bens de capital e de bens de consumo, respectivamente. O símbolo "I" foi trocado por "Y" para designar a renda ou produto. O lucro corrente é representado por "P", o lucro esperado por "P*", o nível de preços por "p" (" $p_{I}$ ” e " $p_{C}$ ” em cada setor), a quantidade produzida por " $q$ " (" $\mathrm{q}_{\mathrm{I}}$ " e " $\mathrm{q}_{\mathrm{C}}$ " em cada setor), o estoque de capital por "K" e a parcela da renda que os agentes demandam na forma líquida por "k". "M" representa a quantidade de moeda, "N" o nível de emprego, "w" o salário per capita e "r" a taxa de juros. "S" é o montante de poupança e "s" a propensão a poupar. 


$$
\begin{aligned}
& p_{I}=w_{I}\left(\frac{d N_{I}}{d q_{I}}\right) \\
& p_{C}=w_{C}\left(\frac{d N_{C}}{d q_{C}}\right) \\
& Y=q_{I} p_{I}+q_{C} p_{C} \\
& Y=P+w N \\
& N=N_{I}+N_{C} \\
& q p_{I}=s Y \\
& r=\frac{P^{*}}{p_{I}} \\
& \frac{p_{I} K}{(M-k Y)}=L(r)
\end{aligned}
$$

As duas primeiras equações descrevem a condição de equilíbrio, no mercado de trabalho, do setor produtor de bens de capital e do setor produtor de bens de consumo, respectivamente, em que o salário real iguala o produto marginal ${ }^{5}$. Embora Meade não explicite funçóes de produção, elas estão implícitas nessas duas equações, dando origem à medida da elasticidade de oferta (supostamente igual nos dois setores), que é dada por $\eta=\frac{d q}{q} \frac{\frac{d N}{d q}}{d\left(\frac{d N}{d q}\right)}$.

A terceira equação descreve a igualdade entre o produto total e a renda e a quarta descreve a decomposição da renda em lucros e salários. Esta dá origem à definição da participação dos lucros na renda, dada por $\frac{P}{Y}=(1-l) \cdot{ }^{6}$

A quinta equação descreve a distribuição do volume total de emprego entre os dois setores e a sexta equação descreve a condição de equilíbrio em que o investimento $\left(I=p_{I} q_{I}\right)$ iguala a poupança $(S=s Y)$.

A sétima equação descreve a igualdade entre a taxa de juros e a eficiência marginal do capital. Meade não explicita os determinantes da taxa de juros, mas implicitamente considera que ela ajusta a oferta de moeda à demanda por moeda, ou seja, é determinada no mercado monetário e não pela igualdade entre investimento e poupança. A eficiência marginal do capital, por sua vez, é descrita como a relação entre o lucro esperado e o
${ }^{5}$ Equivalente, na forma genérica (ou agregada), a $\frac{w}{p}=\left(\frac{d q}{d N}\right)$.

${ }^{6}$ Onde $l=\frac{w N}{Y}$ com $0<l<1$. 
${ }^{7}$ Heller (2001) destaca que não são relaçóes de causalidade, as quais só podem ser inferidas dos argumentos em prosa fornecidos por Meade, já que náo correspondem a qualquer equação do seu sistema, e identifica apenas três relaçóes de causalidade: (i) entre o consumo e a renda; (ii) entre a demanda por moeda (por um lado) e a renda e a taxa de juros (por outro); e (iii) entre a demanda por moeda pelos motivos precaução e especulação (por um lado) e a taxa de juros (por outro). preço corrente de uma unidade de capital, ou seja, $e m c=\frac{P^{*}}{p_{I}}$. Vale ressaltar que o lucro esperado refere-se à renda esperada, em cada período futuro, de uma unidade de bem de capital instalada no presente ao preço $p_{I}$. Para simplificar, Meade supóe que o mesmo lucro é esperado nos próximos períodos e que depende do lucro corrente P. A relação entre ambos é a medida de sensibilidade dos lucros futuros à variação dos lucros correntes, dada por $\pi=\frac{d P^{*}}{P^{*}} \frac{P}{d P}$.

A oitava equação descreve a demanda por moeda através do conceito de preferência pela liquidez, que depende da razão entre o valor do estoque de capital instalado e a quantidade de moeda "ociosa", isto é, a parcela do montante total de moeda que não é demandada pelo motivo transação. A elasticidade da preferência pela liquidez em relação à taxa de juros é dada por $\lambda=\frac{d L(r)}{L(r)} \frac{r}{d r}$.

As oito equações descrevem as condiçôes de equilíbrio e estão referidas a oito incógnitas (quantidades produzidas de bens de capital e de consumo $\left(q_{I}, q_{C}\right)$, seus respectivos preços $\left(p_{I}, p_{C}\right)$, a renda agregada $Y$, o lucro $P$, a taxa de juros $r$ e o volume de emprego $N$ ), duas constantes (o estoque de bens de capital $K$ e a proporção $k$ da renda que as pessoas desejam manter na forma de moeda a qualquer momento para financiar as transaçóes correntes), e três variáveis independentes (a quantidade de moeda $M$, o salário per capita $w$ e a proporçáo poupada da renda s). Assim, dadas as constantes e as variáveis independentes, determina-se o volume de emprego.?

\section{2_As condições de estabilidade do equilíbrio}

Para tratar das condiçôes de estabilidade do equilibrio, Meade sugere partir de um suposto "aumento acidental" (exógeno) nos gastos de bens de capital e de consumo que, por ser acompanhado por um aumento adequado da oferta, não gera pressão sobre preços; ademais, todas as outras variáveis (dependentes e independentes) permanecem inalteradas. As consequências imediatas do aumento do gasto são "(a) a elevação das rendas e, portanto, do montante que as pessoas desejam poupar; (b) o aumento dos lucros e, portanto, das expectativas de lucro futuro, gerando um incentivo para a tomada de empréstimos para investir" (MEADE, 1937, p. 100). Em outras palavras, o aumento inicial e exógeno do dispêndio gera um aumento da pou- 
pança (em decorrência do aumento da renda) e um aumento do investimento (em decorrência do aumento dos lucros). Dependendo da relação entre o aumento da poupança e o do investimento, o sistema poderá estar em equilíbrio estável ou instável.

Segundo a concepção de Meade, se a poupança aumentar mais do que o investimento o sistema estará em equilíbrio estável, pois nesse caso não é possível sustentar o nível mais elevado da renda - ou seja, ela retorna ao seu nível de equilíbrio inicial. No caso contrário (se a poupança aumentar menos do que o investimento), a renda continuará crescendo até encontrar um novo nível de equilíbrio mais alto. Isto, para Meade, significa que o equilíbrio é instável. Esse é o significado de estabilidade solicitado por Frisch.

\section{3_Os testes de estabilidade}

Mas, para além da definição das condições básicas de equilíbrio estável, Meade sugere um teste de estabilidade em que distingue duas políticas bancárias possíveis frente ao "aumento acidental" dos gastos: uma na qual os bancos não alteram a quantidade de moeda (uma das três variáveis independentes) e outra na qual eles não alteram a taxa de juros (que, no entanto, havia definido como variável dependente). Esse exercício é depois complementado com consideraçóes sobre as consequências de mudanças em outras variáveis, sem a suposição inicial do aumento dos gastos (tratadas na segunda seção deste texto). A análise de Meade baseia-se em medidas de elasticidade que estão listadas em seu apêndice matemático, mas cujo cálculo ele não demonstra, a saber: ${ }^{8}$

$\varepsilon_{r}=\frac{\frac{d N}{N}}{\frac{d r}{r}}=-\eta \times \frac{1-l}{l} \times \frac{1}{1-l-\pi}$

$\varepsilon_{M}=\frac{\frac{d N}{N}}{\frac{d M}{M}}=\eta \times \frac{1-l}{l} \times \frac{1}{(1-l)(1+\eta[1-m]+m \lambda)-m \lambda \pi}$

$\varepsilon_{w}=\frac{\frac{d N}{N}}{\frac{d w}{w}}=-\varepsilon_{M}(1-m \lambda[\pi-1])$

$\varepsilon_{s}=\frac{\frac{d N}{d s}}{\frac{N}{s}}=-\frac{(1+\lambda) m}{1+\eta} \times \varepsilon_{M}$ 
${ }^{9}$ Nas palavras do autor, o equilíbrio é estável se, em consequência do aumento do gasto, "for preciso reduzir a taxa de juros... à medida que o emprego aumenta, pois a menos que a taxa de juros caia, qualquer aumento eventual da renda, dos lucros e do emprego estimularia o investimento numa proporção menor do que a poupança, e esta expansão não poderia ser mantida" (MEADE, 1937, p. 100). Por sua vez, o equilíbrio será instável se, em consequência do aumento do gasto, "for preciso elevar a taxa de juros para preservar o equilíbrio à medida que o emprego aumenta, pois isto significa que qualquer aumento eventual da renda, dos lucros e do emprego estimularia o investimento numa proporção maior do que a poupança...” (MEADE, 1937, p. 100).
Vale lembrar que:

$$
\begin{gathered}
\eta=\frac{d q}{q} \frac{\frac{d N}{d q}}{d\left(\frac{d N}{d q}\right)} \begin{array}{l}
\text { é a elasticidade de oferta } \\
\text { (supostamente igual nos } \\
\text { dois setores); }
\end{array} \\
l=\frac{w N}{Y} \quad \begin{array}{l}
\text { é a participação } \\
\text { dos salários na renda; }
\end{array} \\
\pi=\frac{d P^{*}}{P^{*}} \frac{P}{d P} \quad \begin{array}{l}
\text { é a sensibilidade dos } \\
\text { lucros futuros à variação } \\
\text { dos lucros correntes; }
\end{array}
\end{gathered}
$$

$$
\begin{aligned}
m=\frac{M-k Y}{M} & \begin{array}{l}
\text { é a proporção de moeda } \\
\text { "ociosa” em relaçáo à }
\end{array} \\
& \begin{array}{l}
\text { quantidade total de } \\
\text { moeda; }
\end{array}
\end{aligned}
$$

$$
\begin{aligned}
\text { e } \lambda=\frac{d L(r)}{L(r)} \frac{r}{d r} & \begin{array}{l}
\text { é a elasticidade da } \\
\text { preferência pela liquidez } \\
\text { em relação à taxa de juros. }
\end{array}
\end{aligned}
$$

\section{a. 0 teste de estabilidade com juros constantes}

No caso em que, com o aumento dos gastos, os bancos decidem manter a taxa de juros constante, Meade conclui que o equilíbrio será estável se, mantidas inalteradas as variáveis independentes (taxa nominal de salários e proporção poupada da renda), a elevação exógena do gasto provocar pressão pela redução da taxa de juros, pois isso indica que o investimento foi pouco estimulado - o que para o autor representa um equilíbrio estável. Simetricamente, o equilíbrio será instável se for preciso elevar a taxa de juros para que o investimento seja desestimulado. ${ }^{9}$

Para analisar essa relação, Meade utiliza a elasticidade do nível de emprego em relação à taxa de juros dada pela equaçáo (9), em que $\varepsilon_{r}=\frac{\frac{d N}{N}}{\frac{d r}{r}}=-\eta \times \frac{1-l}{l} \times \frac{1}{1-l-\pi}$. Ou seja, supóe que a política escolhida é a que mantém a taxa de juros constante, e ao mesmo tempo usa a elasticidade do nível de emprego em relaçáo à taxa de juros para avaliar as condiçóes de estabilidade do sistema. Vale notar que Meade não explora todas as relaçóes que suas medidas de elasticidade permitiriam explorar, restringindo-se a avaliar se elas são positivas ou negativas, isto é, se o impacto da alteração de uma variável sobre a outra se dá na mesma direção ou na direção contrária, deixando de lado consideraçóes sobre esse impacto ser maior ou menor do que a variação original. Nesse sentido, podese concluir que não há em Meade uma análise quantitativa propriamente dita, mas apenas qualitativa.

Importa ressaltar que mesmo sendo uma análise fundamentalmente qualitativa, Meade nem sempre explica o conteúdo econômico das suas relaçóes 
matemáticas - nem apresenta os passos das relaçóes matemáticas que constrói. No caso em questão, consideramos ser possível sustentar a conclusão de Meade de que o equilíbrio é estável se $\pi<(1-l)$ com base em dois pressupostos: i) a sensibilidade do lucro esperado a mudanças no lucro corrente $(\pi)$ determina o investimento e ii) a participação dos lucros na renda $(1-l)$ determina a propensão a poupar a partir do lucro. Sendo assim, como a estabilidade do equilíbrio exige que o incentivo a investir seja menor do que o incentivo a poupar, temos que o equilíbrio estável exige que $\pi<(1-l)$. Essa condição implica que $\varepsilon_{r}<0$.

\section{b. 0 teste e estabilidade com oferta de moeda constante}

Alternativamente, se ao invés de manterem a taxa de juros constante quando há um aumento dos gastos, os bancos mantiverem o estoque de moeda constante, a condição de estabilidade é menos severa. Nesse caso, o aumento da renda e dos gastos reduzirá a quantidade de moeda "ociosa" em decorrência do aumento da demanda por moeda pelo motivo transação. Isso pressionará pelo aumento da taxa de juros. A regra geral de condição de estabilidade é a mesma (estabilidade quando a poupança se eleva mais do que o investimento e vice-versa), e como o aumento da taxa de juros reduz o incentivo a investir, é menos provável que o investimento cresça mais que a poupança. Ou seja, o equilíbrio é estável se for preciso elevar a oferta de moeda, indicando que a elevação dos juros decorrente do aumento dos gastos reduz o investimento. Por outro lado, se for preciso reduzir a oferta de moeda o equilíbrio é instável, pois a elevação dos juros não teria sido suficiente para reduzir o investimento. Para esse caso, Meade também usa um cálculo de elasticidade, agora do nível de emprego em relação à oferta de moeda, dada pela equação (10) em que

$\varepsilon_{M}=\frac{\frac{d N}{N}}{\frac{d M}{M}}=\eta \times \frac{1-l}{l} \times \frac{1}{(1-l)(1+\eta[1-m]+m \lambda)-m \lambda \pi} \cdot{ }^{10}$

Aqui também se observa que Meade supóe que a política escolhida é a que mantém a oferta de moeda constante, e ao mesmo tempo usa a elasticidade do nível de emprego em relação à oferta de moeda para avaliar as condiçóes de estabilidade do sistema. Para Meade, se essa elasticidade é positiva, ela indica que uma variação da oferta de moeda

$$
\begin{aligned}
& { }^{10} \text { Corrigida para: } \\
& \varepsilon_{M}=\eta \times \frac{1-l}{l} \times \frac{1}{(1-l)[1+(1-m)(\eta-l)+m \lambda-m \lambda \pi]}
\end{aligned}
$$


${ }^{11} \mathrm{E}$ isso só é possível quando o denominador do último termo do lado direito da equação for positivo: usando a equação original de Meade, temos que se

$(1-l)(1+\eta[1-m]+m \lambda)-m \lambda \pi>0$,

então

$\pi<\frac{(1-l)(1+\eta[1-m]+m \lambda)}{m \lambda} \mathrm{e}$,

consequentemente,

$\pi<(1-l)\left(1+\frac{1+\eta[1-m]}{m \lambda}\right)$. provoca uma variação no nível de emprego na mesma direção. ${ }^{11}$

É importante chamar a atenção para o fato de que na suposição anterior (a de que os bancos mantêm a taxa de juros constante), o equilíbrio é estável se $\pi<(1-l)$. Nesse caso, o equilíbrio certamente é estável com uma oferta de moeda constante (já que o aumento dos gastos pressiona pela elevação dos juros, reduzindo o incentivo ao investimento). Mas o equilíbrio pode ser estável quando a oferta de moeda é constante, embora não o seja quando a taxa de juros é
Usando a equação corrigida,

temos que se

$$
(1-l)[1+(1-m)(\eta-l)+m \lambda-m \lambda \pi]>0,
$$

então

$\pi<1+\frac{1+(\eta-l)(1-m)}{m \lambda}$

${ }^{12}$ Observe-se que essas condiçóes permanecem as mesmas para a equação corrigida.

${ }^{13}$ Se $\pi$ determina o investimento e $(1-l)$ determina a poupança, e se a estabilidade do equilíbrio exige que o incentivo a investir seja menor do que o incentivo a poupar, temos que $\pi<(1-l)$ é a condição de estabilidade. constante. Isso ocorre quando o aumento do gasto exige uma elevação da taxa de juros (para desestimular o investimento). Ao considerar a condição de equi-

líbrio dada por $\pi<(1-l)\left(1+\frac{1+\eta[1-m]}{m \lambda}\right)$,

Meade conclui que o equilíbrio é provavelmente mais estável quanto menores forem os valores de $\pi, l, m$ e $\lambda$ e quanto maior for o valor de $\eta .{ }^{12}$ No que diz respeito a $\pi$ e $l$, valem as nossas explicaçóes anteriores. ${ }^{13}$ No que se refere às demais variáveis, sugerimos considerar os seguintes pressupostos:

_ se o montante de moeda "ociosa" é uma pequena proporção da quantidade total de moeda ( $m=\frac{M-k Y}{M}$ pequeno), um aumento na demanda por moeda decorrente do aumento dos gastos não tem como ser suprido pela quantidade de moeda ociosa (que é pequena em relação ao total disponível), o que gera grande pressão sobre os juros, reduzindo o incentivo a investir. O equilíbrio tende a ser estável, conforme alega Meade;

_ se a elasticidade da preferência pela liquidez em relação à taxa 
de juros $\lambda=\frac{d L(r)}{L(r)} \frac{r}{d r}$ é pequena,

o aumento do gasto exige uma grande elevaçáo da taxa de juros para "liberar" liquidez, o que reduz o incentivo a investir e, portanto, o equilíbrio também tende a ser estável, de acordo com a conclusão de Meade;

_ a interpretação sobre o tamanho da elasticidade da oferta não está clara.

$$
\eta=\frac{d q}{q} \frac{\frac{d N}{d q}}{d\left(\frac{d N}{d q}\right)}
$$

Meade afirma que $\eta$ precisa ser grande para que $o$ equilíbrio seja estável, mas $\eta$ grande significa que um aumento do gasto induz um grande aumento no nível de emprego, o que de acordo com a definição de Meade é a característica de um equilíbrio instável. Aqui sugerimos uma interpretação perfeitamente compatível com o ambiente da época, que é o pressuposto de que o crescimento do nível de emprego ocorre a partir de um alto nível de desemprego inicial (e talvez esta seja a razão pela qual Meade discuta outras influências sobre o nível de emprego, tema da nossa próxima seção).

\section{3_Efeitos de outras mudanças exógenas sobre o nível de emprego}

Conforme anunciado, Meade também discute os efeitos de outras mudanças exógenas sobre a demanda por trabalho. As mudanças em questão são (i) uma redução na taxa de juros; (ii) uma elevação da oferta de moeda; (iii) uma redução dos salários e (iv) uma redução da propensão a poupar.

Tal como faz para determinar as condiçóes em que o equilíbrio pode ser considerado estável, aqui também Meade utiliza elasticidades para identificar as condições que devem ser satisfeitas para que o nível de emprego aumente. ${ }^{14}$

\section{1_Efeito da variação dos juros}

Supondo que a taxa de salário nominal $w$ e a proporção da renda a poupar $s$ sejam constantes, e que os bancos reduzem a taxa de juros, que permanece constante neste novo nível, Meade utiliza a elasticidade da demanda por trabalho em relação à taxa de juros dada pela equação (9).
${ }^{14}$ Heller (2001) destaca que, nessa parte do seu artigo, Meade usa a expressão "demanda por trabalho" em vez do termo "emprego", o que permite afirmar que para Meade o nível de emprego não é determinado pelo equilíbrio no mercado de trabalho e sim que depende da demanda por trabalho, e que esta, por sua vez, é influenciada pelas variáveis mencionadas, das quais uma (a taxa de juros) é uma variável dependente no seu modelo de oito equaçóes. 
${ }_{15}$ Aqui também o uso da equação corrigida não altera os resultados teóricos.
O nível de emprego varia inversamente à variação da taxa de juros, o que significa que $\varepsilon_{r}<0$, ou seja, uma redução dos juros tende a elevar o nível de emprego. Para que isso não ocorra (a condição de estabilidade), é preciso que tanto $\eta$ quanto $\pi$ sejam pequenos. Isso condiz com os pressupostos discutidos anteriormente, embora na discussão das condiçóes de estabilidade o ponto de partida tenha sido uma elevação dos gastos e seu efeito sobre o investimento e a poupança, e agora a equação seja usada para discutir o efeito de uma redução da taxa de juros. Meade nada diz sobre $(1-l)$ - a participação dos lucros na renda -, mas é possível considerar que essa variável também tem que ser pequena, pois é um dos componentes da demanda agregada. Finalmente, note-se que ao variar os juros, ele fixa os salários $w$ e a propensão a poupar $s$, mas não tece consideraçóes sobre a oferta de moeda $M$.

\section{2_Efeito da variação da oferta de moeda}

As consideraçóes sobre os efeitos de uma variaçáo da oferta de moeda sobre a demanda por trabalho supóem novamente que o salário nominal $w$ e a proporção da renda poupada s sejam constantes, mas que os bancos aumentem a oferta total de moeda, que permanece constante no novo nível. Meade usa a elasticidade da demanda por trabalho em relação à da oferta de moeda, dada pela equaçáo (10). Como antes, $\varepsilon_{M}>0$ representa a concepção de que uma elevação da quantidade de moeda tende a elevar o nível de emprego. Para que isso não ocorra (a condiçãao de estabilidade), é preciso que $\eta, \pi$ e $m$ sejam pequenos e que $\lambda$ seja grande. Novamente, Meade não fornece explicações de caráter teórico para esses resultados. A explicação que sugerimos para o tamanho pequeno de $\eta$ e $\pi$ são as mesmas de antes. A condiçáo de $\lambda$ grande implica que o desejo de converter ativos não líquidos em moeda é muito sensível à taxa de juros. Ou seja: o aumento da oferta de moeda, que reduz a taxa de juros, libera liquidez suficiente sem reduzir os juros (e, portanto, sem incentivar o investimento). A condição de $m$ pequeno, por sua vez, significa que não há uma grande demanda por liquidez e essa é uma razão adicional para que os juros não tenham que ser reduzidos. Observa-se, mais uma vez, que também aqui Meade nada diz sobre $(1-l)$, a participação dos lucros na renda, mas como antes é possível considerar que essa variável deve ser pequena. Finalmente, notese que ao variar a quantidade de moeda, Meade fixa os salários $w$ e a propensão a poupar $s$, mas não tece consideraçóes sobre a taxa de juros $r{ }^{15}$ 


\section{3_ Efeito da variação dos salários}

Para a análise dos efeitos de uma variação dos salários nominais sobre o nível de emprego, Meade utiliza a medida da elasticidade do emprego em relaçáo ao salário, dada pela equação (11). Nesse caso, ele avalia os resultados de acordo com três alternativas para o valor de $\pi$, a sensibilidade dos lucros futuros à variação dos lucros correntes, quais sejam, $\pi=1, \pi<1$ e $\pi>1$.

\section{a. Efeito da variação dos salários para $\pi=1$}

Meade conclui que se $\pi=1$, uma dada redução na taxa de salário nominal terá o mesmo efeito sobre o aumento do emprego que um aumento, na mesma proporção, da oferta de moeda, já que nesse caso temos $\varepsilon_{w}=-\varepsilon_{M}$. Aqui parece que Meade desconsidera os argumentos de Keynes, pois para Keynes a queda no salário nominal não aumenta o nível de emprego ou do produto, a menos que haja uma variação em algum dos determinantes da demanda efetiva. Conforme destacado por Almeida (2008), segundo Keynes o nível de emprego depende unicamente da demanda efetiva medida em unidades de salários, e sendo essa a soma do consumo provável e do investimento provável, não pode variar se a propensão a consumir, a eficiência marginal do capital ou a taxa de juros (os determinan- tes da demanda efetiva) permanecerem constantes. De fato:

Para refutar a conclusão sumária de que uma redução dos salários nominais aumentaria o emprego "porque reduz o custo de produção", talvez seja útil seguir o curso dos acontecimentos na hipótese mais adequada a esse raciocínio, isto é, que os empresários esperam que a redução dos salários nominais produza esse efeito. Sem dúvida, não é improvável que o empresário individual, vendo diminuir seus próprios custos, comece negligenciando efeitos sobre a demanda de seu produto e atue baseado na hipótese de que será capaz de vender com lucro uma produção maior que a de antes. Se os empresários em geral regularem sua atitude baseando-se nesta expectativa, conseguirão eles na realidade aumentar os seus lucros? Somente se a propensão marginal a consumir da comunidade for igual à unidade, de modo que náo haja lacuna entre o incremento dos rendimentos e o incremento do consumo: ou então se houver um aumento no investimento que corresponda à lacuna existente entre o incremento da renda e o incremento do consumo, o que só acontecerá no caso de a curva das eficiências marginais do capital ter aumentado relativamente à taxa de juros. Desse modo, os resultados obtidos com o aumento de produçâo desanimarâo os empresários, e o emprego voltará, 
outra vez, ao seu nivel anterior, salvo se a propensão marginal a consumir for igual à unidade ou a redução nos salários nominais resultar num aumento da escala das eficiências marginais do capital em relação à taxa de juros $e$, portanto, no montante do investimento. (KEYNES, 1936, p. 250)

$\mathrm{O}$ argumento de Meade se desenvolve com base em um exemplo numérico no qual supõe uma redução de $10 \%$ do salário nominal combinada com uma redução de $10 \%$ na oferta de moeda, sem alteração na taxa de juros. ${ }^{16}$ Meade supõe que a redução dos salários reduz o preço dos bens de capital, ao mesmo tempo que a queda da oferta de moeda - na mesma proporção que a redução dos salários - reduz a demanda por moeda "ociosa". Nesse caso específico, em que se atribui o valor unitário à elasticidade dos lucros esperados em relaçáo aos lucros correntes $(\pi=1)$, para que ocorra aumento do nível de emprego é preciso que haja ou uma redução dos salários ou uma elevação da oferta de moeda, mas nunca uma variaçáo proporcionalmente igual (e na mesma direção) das duas variáveis. ${ }^{17}$

Assim, pode-se afirmar que Meade chega às mesmas conclusóes que Keynes - mas desde que, junto com a queda do salário, haja também a queda de um dos determinantes da demanda efe-
${ }^{16}$ Para uma redução de $10 \%$ do salário nominal combinada com uma redução de $10 \%$ na oferta de moeda, se $\pi=1$ entấo $\varepsilon_{w}=-\varepsilon_{M}$, os efeitos se anularão. Assim, a produção e o emprego permanecem inalterados, o custo primário marginal e o preço de todos os bens caem $10 \%$ (em vista dos $10 \%$ de queda dos salários nominais) e consequentemente a renda nominal cai em 10\% (mas a renda real ou o produto náo se alteram, pois os preços caem). Isso faz com que $10 \%$ menos moeda seja necessária para as transaçóes correntes e, como a oferta total de moeda também foi reduzida em $10 \%$, a oferta de moeda "ociosa" também cai em 10\%. Mas, como o preço dos bens de capital também cai em $10 \%$, a relaçáo entre o valor dos ativos não líquidos e o montante de moeda "ociosa" não muda, e consequentemente não há alteração na preferência pela liquidez e a taxa de juros fica inalterada. Além disso, a poupança nominal cai em $10 \%$ devido à queda de $10 \%$ na renda nominal, e o investimento nominal também cai $10 \%$ se os lucros esperados caírem na mesma proporção; como a taxa de juros náo se altera, e o preço da oferta de bens de capital e o produto nominal esperado sobre eles caem $10 \%$, não há incentivo para mudanças no valor do investimento real e, portanto, o valor nominal do investimento cai à mesma taxa que o preço dos bens de capital.

${ }^{17}$ Heller (2001) nota que a consideraçáo simultânea dos efeitos de uma variaçáo nos salários e na oferta de moeda inexiste nos manuais de macroeconomia. Nesses, a taxa de juros se reduz se houver um aumento da oferta de moeda para uma demanda por moeda inalterada (ou se houver uma reduçáo da demanda por moeda para uma dada oferta de moeda). Variações dos salários não têm influência sobre a taxa de juros. No argumento de Meade, no entanto (mas apenas implicitamente), há a sugestão de que a redução dos salários também reduz a taxa de juros, pois a reduçáo dos salários reduz os preços e a renda nominal, reduzindo, por consequência, a demanda por moeda. 
tiva que determina o emprego (no caso, queda da taxa de juros). ${ }^{18}$

\section{b. Efeito da variação dos salários para $\pi<1$}

O significado de $\pi<1$ é que uma queda de, por exemplo, $10 \%$ nos lucros correntes, fará o lucro futuro esperado cair menos que $10 \%$. Nesse caso, segundo Meade, uma queda de $10 \%$ no salário nominal, que é acompanhada de uma redução do preço de oferta nominal corrente dos bens de capital $\left(p_{\mathrm{I}}\right)$ e do lucro nominal corrente $(P)$, leva a um aumento da relação entre os lucros esperados e o preço de oferta dos bens de capital (eficiência marginal do capital) e, portanto, encoraja o investimento. Assim, nessas condições, uma redução do salário nominal é mais eficaz em aumentar o emprego que um aumento na mesma proporção da oferta de moeda, já que agora, além da queda na taxa de juros, teremos também o aumento da eficiência marginal do capital, que faz com que o investimento aumente ainda mais. Também nesse caso podese afirmar que Meade chega às mesmas conclusóes que Keynes - mas desde que junto com a queda do salário haja também a elevação da eficiência marginal do capital, um dos determinantes da demanda efetiva que por sua vez determina o nível de emprego. ${ }^{19}$

\section{c. Efeito da variação dos salários para $\pi>1$}

Inversamente, se $\pi>1$ a queda no lucro nominal esperado é maior que a queda, em proporção, dos lucros nominais correntes. Nesse caso, uma redução do salário nominal tem um efeito menor sobre o nível de emprego do que uma elevação (na mesma proporção) da oferta de moeda. Em outras palavras, a redução do salário nominal leva a uma menor eficiência marginal do capital. Assim, apesar de a taxa de juros cair, a eficiência marginal

\footnotetext{
${ }^{18} \mathrm{O}$ raciocínio de Meade equivale ao chamado "efeito Keynes", que se refere ao impacto da reduçấo de preços (causada pela reduçáo dos salários) sobre a demanda por moeda pelo motivo transação, que diminui em decorrência da menor necessidade de moeda, já que os preços se

\author{
reduziram. Para uma dada \\ oferta de moeda, a escassez \\ relativa da demanda por \\ moeda provoca reduçáo da \\ taxa de juros e, portanto, \\ elevação de investimentos e \\ do nível de emprego. \\ ${ }^{19}$ Em termos algébricos, e \\ de acordo com a formulaçáo \\ da elasticidade da demanda
}

por trabalho em relação aos salários dada por $\varepsilon_{w}=-\varepsilon_{M}(1-m \lambda[\pi-1])$, a suposição $\pi<1$ resulta que, em valores absolutos, $\left|\varepsilon_{w}\right|<\left|\varepsilon_{M}\right|$. Esse resultado, que contradiz a argumentação de Meade, considera: (i) que $\lambda<0$, pois a preferência pela liquidez varia na direção
} oposta da variaçáo da taxa de juros, e (ii) que $\pi>0$, pois os lucros esperados variam na mesma direção que a variação dos lucros correntes (neste caso, portanto, $0<\pi<1$ ). Além disso, $0<m<1$. 
do capital também se reduz e, portanto, o aumento no emprego, dado o aumento no investimento, é menor.

\section{4_Efeito da variação da propensão a poupar}

Finalmente, para avaliar os efeitos de uma variação na propensão a poupar sobre o nível de emprego, Meade utiliza a medida da elasticidade do emprego em relação à propensáo a poupar dada pela equaçáo (12), e supóe que a taxa de salário nominal $w$ e a oferta de moeda $M$ são constantes. Como vimos, $\varepsilon_{M}$ é positiva e, portanto, $\varepsilon_{s}$ deve ser negativa. Como a propensão a consumir é o complementar da propensão a poupar, pode-se dizer que, com esta elasticidade, Meade analisa o terceiro componente da demanda efetiva (lembrando que os anteriores são a taxa de juros e a eficiência marginal do capital). Mas, como nos demais casos, Meade restringe a análise à avaliação da direção do impacto da variação de uma dada variável sobre o nível de emprego, sem discutir o tamanho desse impacto. Aqui, ao menos, ele reconhece que uma queda na proporção a poupar (que equivale a uma elevação da propensão a consumir) levará a um aumento do emprego, e explicita esse argumento de forma muito clara:
Uma redução da proporção poupada da renda aumenta o gasto em consumo; haverá então um aumento do gasto total e do emprego, a não ser que o investimento caia na mesma proporção que o aumento do consumo. Mas o investimento só cai se houver uma elevação da taxa de juros, $e$ a taxa de juros só se eleva se houver uma redução da oferta de moeda "ociosa". Mas, para uma dada oferta total de moeda, a oferta de moeda "ociosa" só cai se houver um aumento no gasto total, provocando um aumento da demanda por moeda para financiar as transaçöes correntes. Portanto, a taxa de juros não pode se elevar o suficiente para reduzir o investimento na mesma proporção em que o gasto em consumo se elevou (MEADE, 1937, p. 104).

\section{4_Conclusão - uma síntese da contribuição de Meade}

A partir do que foi exposto anteriormente, verifica-se que Meade constrói um sistema de oito equaçóes para determinar as condiçóes de equilíbrio de curto prazo de uma economia em concorrência perfeita, fechada e sem governo, produtora de bens de consumo e de bens de investimento. Essas oito equaçóes também servem para determinar as condi- 
ções de estabilidade desse equilíbrio, em que estabilidade é entendida como a situação em que o sistema, depois de submetido a um choque de demanda (aumento exógeno do dispêndio), retorna à posição inicial. $\mathrm{Na}$ formulação de $\mathrm{Me}$ ade, isso significa que o estímulo que o choque de demanda exerce sobre o investimento deve ser inferior ao que exerce sobre a poupança. Essa é a definiçãoo de estabilidade solicitada por Frisch.

A análise de Meade é mais qualitativa do que quantitativa, e ele nem sempre explicita o raciocínio econômico que sustenta suas conclusóes. Procuramos complementar essas lacunas ao longo do texto, e aqui apresentamos um resumo das principais relaçóes teóricas propostas, mas de forma mais genérica do que anteriormente: (i) além da taxa de juros e da eficiência marginal do capital, o lucro esperado também determina o incentivo a investir; (ii) o montante de moeda "ociosa" em relação à quantidade total de moeda determina o impacto que uma variação na demanda por moeda, decorrente de uma variação dos gastos, tem sobre a taxa de juros e, consequentemente, sobre o incentivo a investir; (iii) a elasticidade da preferência pela liquidez em relaçáo à taxa de juros permite que uma variação da oferta de moeda altere a liquidez do sistema sem afetar a taxa de juros; e (iv) o efeito da variação dos salários sobre o nível de emprego (em comparação com o efeito de uma política monetária expansiva) depende do valor da elasticidade dos lucros esperados em relação aos lucros correntes.

A análise de Meade prioriza a reação do sistema bancário (que decide manter inalterada a taxa de juros ou, alternativamente, a oferta de moeda). A sensibilidade dos lucros esperados à variação dos lucros presentes é a variávelchave e original da sua contribuição, e o papel destacado das expectativas $\pi$ esclarece porque os intérpretes de Meade consideram que seu modelo se assemelha muito mais ao livro de Keynes do que as contribuiçóes de Harrod (1937) e de Hicks (1937). Em particular, destaca-se que na análise de Meade a redução dos salários pode ter efeito positivo sobre o nível de emprego desde que haja também a queda de um dos determinantes da demanda efetiva que determina o emprego (queda da taxa de juros), e/ou a elevação da eficiência marginal do capital - em ambos os casos, sempre dependendo do valor da elasticidade dos lucros esperados em relação aos lucros correntes. 


\section{Referências Bibliográficas}

ALANEZ, E. L. (2001). El modelo algebraico de Meade (1937): El sistema económico simplificado de Keynes. Investigación Económica, v. 61, n. 238, octubrediciembre, p. 69-108.

ALMEIDA, R. 6. (2008). James Meade e a Teoria Geral do Emprego, dos Juros e da Moeda. Monografia de Conclusão de Curso. Departamento de Economia. Unesp, Araraquara. Mimeo, 2008.

Assous, M. (2004) Le modèle keynésien de Meade (1937): première analyse dynamique du sous-emploi? Conferencia " $\mathrm{L} a$ réception de la Théorie Générale 1936-1939", Association pour le Developpement des Etudes Keynesiennes - ADEK, Sceaux, França, outubro de 2004.

\section{CHAMPERNOWNE, David}

Gawen (1935-36): Unemployment, Basic and Monetary: the classical analysis and the keynesian.

Review of Economic Studies, v. 3, 1935-36, p. 201-216.
DARITY, W. E COTTRELL, A. F. (1987): Meade's 'General Theory' model: a geometric reprise. Journal of Money, Credit and Banking, v. 18, n. 2, p. 210-21. HARROD, R. F. (1937) Mr. Keynes and traditional theory. Econometrica, v. 5, janeiro 1937, p. 74-86.

HELLER, C. (2001) O modelo simplificado de A Teoria Geral do Emprego, dos Juros e da Moeda segundo James Meade. Anais do VI Encontro Nacional de Economia Politica (SEP), São Paulo. 2001.

HELLER, C. (2007) Hicks, a teoria geral e a teoria geral generalizada. Economia (ANPEC), v. 8, n. 3, p. 401-436, setembrol dezembro.

HICKS, J. R. (1937) Mr. Keynes and the 'classics': a suggested interpretation. Econometrica, v. 5 , abril 1937, p. 147-159.

KEYNES, J. M. (1936) A teoria geral do emprego, do juro e da moeda. Nova Cultural, São Paulo, 1996
MEADE, J. E. (1937): A simplified model of Mr. Keynes' system. Review of Economic Studies, v. 4, fevereiro, p. 98-107.

RAPPOPORT, P. (1992): Meade's General Theory model: stability and the role of expectations. Journal of Money, Credit and Banking, v. 24, p. 356-69.

REDDAWAY, W.B. (1936): The General Theory of Employment, Interest and Money. Economic Record, v. 12, junho, p. 28-36.

YOUNG, Warren (1987).

Interpreting Keynes: The IS/LM Enigma, Boulder, Colorado: Westview Press; Oxford: Basil Blackwell.

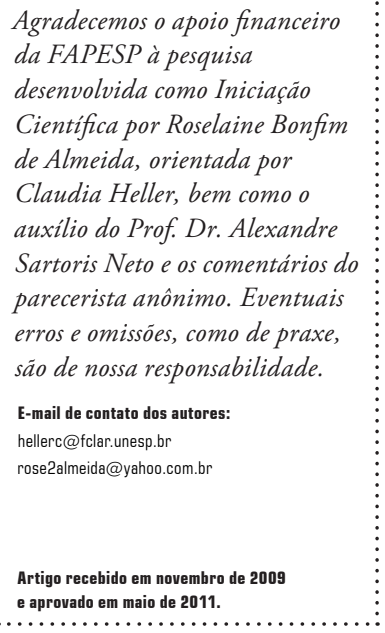


Anexos Matemáticos

Anexo I
Cálculo das diferenciações das relações básicas do modelo de Meade

$$
\begin{aligned}
& \frac{d p_{I}}{p_{I}}=\frac{d w_{I}}{w_{I}}+\frac{d q_{I}}{q_{I}} \cdot \frac{1}{\eta} \\
& \frac{d p_{C}}{p_{C}}=\frac{d w_{C}}{w_{C}}+\frac{1}{\eta} \cdot \frac{d q_{C}}{q_{C}} \\
& \frac{d Y}{Y}=s \cdot\left(\frac{d p_{I}}{p_{I}}+\frac{d q_{I}}{q_{I}}\right)+(1-s) \cdot\left(\frac{d p_{C}}{p_{C}}+\frac{d q_{C}}{q_{C}}\right) \\
& \frac{d Y}{Y}=(1-l) \frac{d P}{P}+l\left(\frac{d w}{w}+\frac{d N}{N}\right) \\
& \frac{d N}{N}=\frac{s}{l} \frac{d q_{I}}{q_{I}}+\frac{(1-s)}{l} \frac{d q_{C}}{q_{C}} \\
& \frac{d s}{s}+\frac{d Y}{Y}=\frac{d q_{I}}{q_{I}}+\frac{d p_{I}}{p_{I}} \\
& \frac{d r}{r}+\frac{d p_{I}}{p_{I}}=\pi \frac{d P}{P} \\
& m \lambda \frac{d r}{r}=m \frac{d p_{I}}{p_{I}}-\frac{d M}{M}+(1-m) \frac{d Y}{Y}
\end{aligned}
$$


Para as equaçóes (1a) e (2a), diferenciamos a expressão genérica $p=w\left(\frac{d N}{d q}\right)$ $\frac{d p}{p}=\frac{d w}{w} \frac{w}{p}\left(\frac{d N}{d q}\right)+w \frac{d\left(\frac{d N}{d q}\right)}{\frac{d N}{d q}} \frac{\frac{d N}{d q}}{p}$, onde $p=w\left(\frac{d N}{d q}\right)$ e $\frac{w}{p}=\frac{1}{\left(\frac{d N}{d q}\right)}$.

Substituindo $\frac{w}{p}=\frac{1}{\left(\frac{d N}{d q}\right)}$ no $1^{\circ}$ termo do lado direito de $\frac{d p}{p}=\frac{d w}{w} \frac{w}{p}\left(\frac{d N}{d q}\right)+w \frac{d\left(\frac{d N}{d q}\right)}{\frac{d N}{d q}} \frac{\frac{d N}{d q}}{p}$, $\operatorname{temos} \frac{d p}{p}=\frac{d w}{w}+w \frac{d\left(\frac{d N}{d q}\right)}{\frac{d N}{d q}} \frac{d N}{d q}$

Rearranjando: $\frac{d p}{p}=\frac{d w}{w}+w \frac{d\left(\frac{d N}{d q}\right) \frac{d N}{d q}}{\frac{d N}{d q}} \frac{d w}{w}+w \cdot \frac{d N}{d q} \cdot d\left(\frac{d N}{d q}\right) \cdot \frac{d q}{d N} \cdot \frac{1}{p}$ A $1^{\text {a }}$ parte do $2^{\mathrm{o}}$ termo do lado direito é o custo marginal $w \cdot \frac{d N}{d q}$ que, em equilíbrio, é
igual ao preço $p$.

Fazendo a devida substituição, temos $\frac{d p}{p}=\frac{d w}{w}+p \cdot d\left(\frac{d N}{d q}\right) \cdot \frac{d q}{d N} \cdot \frac{1}{p}=\frac{d w}{w}+d\left(\frac{d N}{d q}\right) \cdot \frac{d q}{d N}$.

Multiplicando e dividindo o segundo termo do lado direito por $q: \frac{d p}{p}=\frac{d w}{w}+\frac{d q}{q} \cdot \frac{q}{d N} \cdot d\left(\frac{d N}{d q}\right)$ 
Considerando que a elasticidade da oferta é $\eta=\frac{\frac{d q}{q}}{\frac{d\left(\frac{d N}{d q}\right)}{\frac{d N}{d q}}}$, temos $\frac{1}{\eta}=\frac{q}{d N} \cdot d\left(\frac{d N}{d q}\right)$.

O lado direito de $\frac{1}{\eta}$ corresponde aos dois últimos termos do lado direito de $\frac{d p}{p}=\frac{d w}{w}+\frac{d q}{q} \cdot \frac{q}{d N} \cdot d\left(\frac{d N}{d q}\right)$

Substituindo, temos $\frac{d p}{p}=\frac{d w}{w}+\frac{d q}{q} \cdot \frac{1}{\eta}$.

Para a equação (3a) usamos a equação (3): $Y=p_{I} q_{I}+p_{C} q_{C}$

$\frac{d Y}{Y}=\frac{d\left(p_{I} q_{I}\right)}{p_{I} q_{I}} \cdot \frac{p_{I} q_{I}}{Y}+\frac{d\left(p_{C} q_{C}\right)}{p_{C} q_{C}} \cdot \frac{q_{C} p_{C}}{Y}=\frac{p_{I} \cdot d q_{I}+q_{I} \cdot d p_{I}}{p_{I} q_{I}} \cdot \frac{p_{I} q_{I}}{Y}+\frac{p_{C} \cdot d q_{C}+q_{C} \cdot d p_{C}}{p_{C} q_{C}} \cdot \frac{q_{C} p_{C}}{Y}$

Simplificando, e considerando que ${ }_{s}=\frac{p_{I} q_{I}}{Y}$ e que $1-s=\frac{p_{C} q_{C}}{Y}$,

$\frac{d Y}{Y}=\left(\frac{p_{I} \cdot d q_{I}}{p_{I} q_{I}}+\frac{q_{I} \cdot d p_{I}}{p_{I} q_{I}}\right) \cdot s+\left(\frac{p_{C} \cdot d q_{C}}{p_{C} q_{C}}+\frac{q_{C} \cdot d p_{C}}{p_{C} q_{C}}\right)(1-s)$.

Simplificando novamente: $\frac{d Y}{Y}=\left(\frac{d q_{I}}{q_{I}}+\frac{d p_{I}}{p_{I}}\right) \cdot s+\left(\frac{d q_{C}}{q_{C}}+\frac{d p_{C}}{p_{C}}\right) \cdot(1-s)$.

Para a equação (4a) usamos a equação (4): $Y=P+w N$

$\frac{d Y}{Y}=\frac{d P}{P} \cdot \frac{P}{Y}+\frac{d(w N)}{w N} \cdot \frac{w N}{Y}$, onde $l=\frac{w N}{Y}$ e $(1-l)=1-\frac{w N}{Y}=\frac{P}{Y}$

Portanto: $\frac{d Y}{Y}=(1-l) \frac{d P}{P}+l\left(\frac{d w}{w}+\frac{d N}{N}\right)$. 
Para a equação (5a) usamos a equação (5): $N=N_{I}+N_{C}$

$\frac{d N}{N}=\frac{d N_{I}}{N_{I}} \frac{N_{I}}{N}+\frac{d N_{C}}{N_{C}} \frac{N_{C}}{N}=\frac{d N_{I}}{N}+\frac{d N_{C}}{N}$

Multiplicando e dividindo o $1^{\circ}$ termo do lado direito de $\frac{d N}{N}=\frac{d N_{I}}{N}+\frac{d N_{C}}{N}$ por $q_{1}$, w e $d q_{I}$ e o $2^{\mathrm{o}}$ termo do lado direito de $\frac{d N}{N}=\frac{d N_{I}}{N}+\frac{d N_{C}}{N}$ por $q_{c}, w$, e $d q_{c}$, temos $\frac{d N}{N}=\frac{d N_{I}}{N} \frac{q_{I}}{q_{I}} \frac{w}{w} \frac{d q_{I}}{d q_{I}}+\frac{d N_{C}}{N} \frac{q_{C}}{q_{C}} \frac{w}{w} \frac{d q_{C}}{d q_{C}}$.

Rearranjando:

$\frac{d N}{N}=\frac{d N_{I}}{N} \frac{q_{I}}{q_{I}} \frac{w}{w} \frac{d q_{I}}{d q_{I}}+\frac{d N_{C}}{N} \frac{q_{C}}{q_{C}} \frac{w}{w} \frac{d q_{C}}{d q_{C}}=\frac{q_{I} w d N_{I}}{w N} \frac{1}{d q_{I}} \frac{d q_{I}}{q_{I}}+\frac{q_{C} w d N_{C}}{w N} \frac{1}{d q_{C}} \frac{d q_{C}}{q_{C}}$ $\frac{d N}{N}=\frac{q_{I} w \frac{d N_{I}}{d q_{I}}}{w N} \frac{d q_{I}}{q_{I}}+\frac{q_{C} w \frac{d N_{C}}{d q_{C}}}{w N} \frac{d q_{C}}{q_{C}}=\frac{q_{I} p_{I}}{w N} \frac{d q_{I}}{q_{I}}+\frac{q_{C} p_{C}}{w N} \frac{d q_{C}}{q_{C}}$,

onde (genericamente) $w \frac{d N}{d q}=p$

Divida o numerador e o denominador de cada termo de $\frac{d N}{N}=\frac{q_{I} p_{I}}{w N} \frac{d q_{I}}{q_{I}}+\frac{q_{C} p_{C}}{w N} \frac{d q_{C}}{q_{C}}$ por $Y$ e considere que $s=\frac{p_{I} q_{I}}{Y}, 1-s=\frac{p_{C} q_{C}}{Y}, l=\frac{w N}{Y}$ e $1-l=1-\frac{w N}{Y}=\frac{P}{Y}$.

Portanto: $\frac{d N}{N}=\frac{\frac{q_{I} p_{I}}{Y}}{\frac{w N}{Y}} \frac{d q_{I}}{q_{I}}+\frac{\frac{q_{C} p_{C}}{Y}}{\frac{w N}{Y}} \frac{d q_{C}}{q_{C}}=\frac{s}{l} \frac{d q_{I}}{q_{I}}+\frac{(1-s)}{l} \frac{d q_{C}}{q_{C}}$.

Para a equação (6a) usamos a equação (6):

$$
s Y=q_{I} p_{I} \quad \frac{d(s Y)}{s Y}=\frac{d\left(q_{I} p_{I}\right)}{q_{I} p_{I}} \quad \frac{d s \cdot Y+s \cdot d Y}{s Y}=\frac{d q_{I} \cdot p_{I}+q_{I} \cdot d p_{I}}{q_{I} p_{I}}=\frac{d q_{I} \cdot p_{I}}{q_{I} p_{I}}+\frac{q_{I} \cdot d p_{I}}{q_{I} p_{I}}
$$


Portanto: $\frac{d s}{s}+\frac{d Y}{Y}=\frac{d q_{I}}{q_{I}}+\frac{d p_{I}}{p_{I}}$.

Para a equação (7a) usamos a equação (7):

$r=\frac{P^{*}}{p_{I}} \Rightarrow r \cdot p_{I}=P^{*}$

$\frac{d\left(r \cdot p_{I}\right)}{r \cdot p_{I}}=\frac{d P^{*}}{P^{*}} \Rightarrow \frac{d r \cdot p_{I}}{r \cdot p_{I}}+\frac{r \cdot d p_{I}}{r \cdot p_{I}}=\frac{d r}{r}+\frac{d p_{I}}{p_{I}}=\frac{d P^{*}}{P^{*}}$

Considerando que $\pi=\frac{\frac{d P^{*}}{P^{*}}}{\frac{d P}{P}}$, temos que $\frac{d P^{*}}{P^{*}}=\pi \frac{d P}{P}$.

Substituindo, chegamos a $\frac{d r}{r}+\frac{d p_{I}}{p_{I}}=\pi \frac{d P}{P}$.

Para a equação (8a) usamos a equação (8): $L(r)=\frac{p_{I} K}{M-k Y}$ e as definiçóes $m=\frac{M-k Y}{M}$ e $\lambda=\frac{\frac{d L(r)}{L(r)}}{\frac{d r}{r}}$.

Diferenciando em função das três variáveis endógenas $\left(p_{I}, M\right.$ e $\left.Y\right)$ :

$$
\begin{aligned}
& d L(r)=\frac{K}{M-k Y} d p_{I}-\frac{p_{I} K}{(M-k Y)^{2}} d M+\frac{k p_{I} K}{(M-k Y)^{2}} d Y \\
& \frac{d L(r)}{L(r)}=\frac{\frac{K}{M-k Y} d p_{I}}{L(r)}-\frac{\frac{p_{I} K}{(M-k Y)^{2}} d M}{L(r)}+\frac{\frac{k p_{I} K}{(M-k Y)^{2}} d Y}{L(r)}
\end{aligned}
$$

Substituindo $L(r)=\frac{p_{I} K}{M-k Y}$ no lado direito:

$\frac{d L(r)}{L(r)}=\frac{\frac{K}{M-k Y} d p_{I}}{\frac{p_{I} K}{M-k Y}}-\frac{\frac{p_{I} K}{(M-k Y)^{2}} d M}{\frac{p_{I} K}{M-k Y}}+\frac{\frac{k p_{I} K}{(M-k Y)^{2}} d Y}{\frac{p_{I} K}{M-k Y}}$. 
Simplificando: $\frac{d L(r)}{L(r)}=\frac{d p_{I}}{p_{I}}-\frac{1}{M-k Y} d M+\frac{k}{M-k Y} d Y$,

de (8) temos que $L(r)=\frac{p_{I} K}{M-k Y}=\frac{\frac{p_{I} K}{M}}{\frac{M-k Y}{M}}=\frac{\frac{p_{I} K}{M}}{m}=\frac{p_{I} K}{m M} \Rightarrow M-k Y=m M$.

Substituindo $M-k Y=m M$ em $\frac{d L(r)}{L(r)}=\frac{d p_{I}}{p_{I}}-\frac{1}{M-k Y} d M+\frac{k}{M-k Y} d Y$, temos $\frac{d L(r)}{L(r)}=\frac{d p_{I}}{p_{I}}-\frac{1}{m M} d M+\frac{k}{m M} d Y=\frac{d p_{I}}{p_{I}}-\frac{1}{m} \frac{d M}{M}+\frac{k}{m M} d Y$

Multiplicando por $m: \quad m \frac{d L(r)}{L(r)}=m \frac{d p_{I}}{p_{I}}-m \frac{1}{m} \frac{d M}{M}+m \frac{k}{m M} d Y=m \frac{d p_{I}}{p_{I}}-\frac{d M}{M}+\frac{k}{M} d Y$.

Sabemos que $\lambda=\frac{\frac{d L(r)}{L(r)}}{\frac{d r}{r}} \Rightarrow \lambda \frac{d r}{r}=\frac{d L(r)}{L(r)} \Rightarrow m \lambda \frac{d r}{r}=m \frac{d L(r)}{L(r)}$.

Sabemos que $m=\frac{M-k Y}{M} \Rightarrow m=\frac{M}{M}-\frac{k Y}{M}=1-\frac{k Y}{M} \Rightarrow{ }_{1-m}=\frac{k Y}{M} \Rightarrow \frac{1-m}{Y}=\frac{k}{M}$.

Substituindo $m \lambda \frac{d r}{r}=m \frac{d L(r)}{L(r)}$ e $\frac{1-m}{Y}=\frac{k}{M}$ em $m \frac{d L(r)}{L(r)}=m \frac{d p_{I}}{p_{I}}-\frac{d M}{M}+\frac{k}{M} d Y$, temos $m \lambda \frac{d r}{r}=m \frac{d p_{I}}{p_{I}}-\frac{d M}{M}+\frac{(1-m)}{Y} d Y$.

Portanto: $m \lambda \frac{d r}{r}=m \frac{d p_{I}}{p_{I}}-\frac{d M}{M}+(1-m) \frac{d Y}{Y}$. 
Anexos Matemáticos

Anexo II

\section{Cálculo da elasticidade dada pela equação (9)}

$\varepsilon_{r}=\frac{\frac{d N}{N}}{\frac{d r}{r}}=-\eta \times \frac{1-l}{l} \times \frac{1}{1-l-\pi}$

Partindo das equaçóes (5a) e (7a), em que $\frac{d N}{N}=\frac{s}{l} \frac{d q_{I}}{q_{I}}+\frac{(1-s)}{l} \frac{d q_{C}}{q_{C}}$ e $\frac{d r}{r}=\pi \frac{d P}{P}-\frac{d p_{I}}{p_{I}}$, respectivamente, temos $\varepsilon_{r}=\frac{\frac{d N}{N}}{\frac{d r}{r}}=\frac{\frac{s}{l} \frac{d q_{I}}{q_{I}}+\frac{(1-s)}{l} \frac{d q_{C}}{q_{C}}}{\pi \frac{d P}{P}-\frac{d p_{I}}{p_{I}}}$.

Nas equações (1a) e (2a) supomos que os salários não variam (isto é, $\frac{d w_{I}}{w_{I}}=\frac{d w_{C}}{w_{C}}=0$ ), de modo que de (1a) $\frac{d p_{I}}{p_{I}}=\frac{d w_{I}}{w_{I}}+\frac{d q_{I}}{q_{I}} \cdot \frac{1}{\eta}$, temos $\left(\frac{d p_{I}}{p_{I}}\right) \eta=\frac{d q_{I}}{q_{I}}$, e de (2a) $\frac{d p_{C}}{p_{C}}=\frac{d w_{C}}{w_{C}}+\frac{1}{\eta} \cdot \frac{d q_{C}}{q_{C}}$, temos $\left(\frac{d p_{C}}{p_{C}}\right) \eta=\frac{d q_{C}}{q_{C}}$.

Substituindo em $\varepsilon_{r}=\frac{\frac{d N}{N}}{\frac{d r}{r}}=\frac{\frac{s}{l} \frac{d q_{I}}{q_{I}}+\frac{(1-s)}{l} \frac{d q_{C}}{q_{C}}}{\pi \frac{d P}{P}-\frac{d p_{I}}{p_{I}}}$, temos $\varepsilon_{r}=\frac{\frac{d N}{N}}{\frac{d r}{r}}=\frac{\frac{s}{l}\left(\frac{d p_{I}}{p_{I}}\right) \eta+\frac{(1-s)}{l}\left(\frac{d p_{C}}{p_{C}}\right) \eta}{\pi \frac{d P}{P}-\frac{d p_{I}}{p_{I}}}$.

Simplificando, temos $\varepsilon_{r}=\frac{\frac{d N}{N}}{\frac{d r}{r}}=\frac{\frac{\eta}{l}\left[s\left(\frac{d p_{I}}{p_{I}}\right)+(1-s)\left(\frac{d p_{C}}{p_{C}}\right)\right]}{\pi \frac{d P}{P}-\frac{d p_{I}}{p_{I}}}$. 
Supondo que os preços variam na mesma proporção nos dois

setores, chegamos a $\varepsilon_{r}=\frac{\frac{d N}{N}}{\frac{d r}{r}}=\frac{\eta}{l} \frac{\left[\frac{d p}{p}\right]}{\pi \frac{d P}{P}-\frac{d p}{p}}$ e, supondo que os preços

variam na mesma proporção que os lucros (de modo que $\frac{d p}{p}=\frac{d P}{P}$ ), temos

$\varepsilon_{r}=\frac{\frac{d N}{N}}{\frac{d r}{r}}=\frac{\eta}{l} \frac{\left[\frac{d p}{p}\right]}{\pi \frac{d p}{p}-\frac{d p}{p}}=\frac{\eta}{l} \frac{\left[\frac{d p}{p}\right]}{\frac{d p}{p}(\pi-1)}=\frac{\frac{d N}{N}}{\frac{d r}{r}}=\frac{\eta}{l} \frac{1}{(\pi-1)}$.

Finalmente, multiplicando e dividindo por $(1-l)$, temos

$\varepsilon_{r}=\frac{\frac{d N}{N}}{\frac{d r}{r}}=\frac{\eta}{l} \frac{(1-l)}{(\pi-1)(1-l)}=-\frac{\eta}{l} \frac{(1-l)}{-\pi+\pi l+1-l}$.

Para chegar ao resultado de Meade, é preciso desprezar $\pi l$ no denominador, de modo que $\varepsilon_{r}=\frac{\frac{d N}{N}}{\frac{d r}{r}}=-\frac{\eta}{l} \frac{(1-l)}{-\pi+\pi l+1-l}=-\frac{\eta}{l} \times(1-l) \times \frac{1}{1-l-\pi}$.

Vale ressaltar que Meade não desenvolve esse cálculo nem fornece quaisquer indicações de argumentos que sustentariam o procedimento de desprezar o termo $\pi l$ no denominador. Tradicionalmente, isso poderia ser desprezado se tanto $\pi$ quanto $l$ fossem muito pequenos. Vimos que a condiçâo de estabilidade é dada por $\pi<(1-l)$ - Lembrando que $l$ representa a proporção dos salários na renda, é razoável supor que $0<l<1$ e, portanto, em condiçóes de estabilidade, é possível justificar o procedimento de desprezar $\pi l$. O mesmo se aplica à discussão que trata do efeito da variação dos juros sobre o nível de emprego, na qual a conclusão é a de que $\pi$ pequeno é uma das condiçóes de estabilidade - embora sem referências a $(1-l)$. Finalmente, observe-se que no restante do artigo Meade usa outras elasticidades, dispensando as consideraçôes sobre a grandeza de $\pi$ e de $l$ para justificar o procedimento de desprezar $\pi l$. 
ANEXOS MATEMÁTICOS

ANEXO III

\section{Cálculo da elasticidade dada pela equação (10)}

$\varepsilon_{M}=\frac{\frac{d N}{N}}{\frac{d M}{M}}=\eta \times \frac{1-l}{l} \times \frac{1}{(1-l)(1+\eta[1-m]+m \lambda)-m \lambda \pi}$

Partindo das equaçóes (5a) e (8a), em que $\frac{d N}{N}=\frac{s}{l} \frac{d q_{I}}{q_{I}}+\frac{(1-s)}{l} \frac{d q_{C}}{q_{C}}$ e $\frac{d M}{M}=m \frac{d p_{I}}{p_{I}}+(1-m) \frac{d Y}{Y}-m \lambda \frac{d r}{r}, \quad\left(\right.$ pois $\left.\quad m \lambda \frac{d r}{r}=m \frac{d p_{I}}{p_{I}}-\frac{d M}{M}+(1-m) \frac{d Y}{Y}\right)$, respectivamente, temos $\varepsilon_{M}=\frac{\frac{s}{l}\left(\frac{d p_{I}}{p_{I}}-\frac{d w_{I}}{w_{I}}\right) \eta+\frac{(1-s)}{l}\left(\frac{d p_{C}}{p_{C}}-\frac{d w_{C}}{w_{C}}\right) \eta}{m \frac{d p_{I}}{p_{I}}+(1-m) \frac{d Y}{Y}-m \lambda \frac{d r}{r}}$.

Como no cálculo anterior, supondo que os salários não variam (de modo que $\frac{d w_{I}}{w_{I}}=\frac{d w_{C}}{w_{C}}=0$ ), e que os preços variam na mesma proporção nos dois setores (de modo que $\frac{d p_{I}}{p_{I}}=\frac{d p_{C}}{p_{C}}$ ), temos $\varepsilon_{M}=\frac{\frac{s}{l}\left(\frac{d p}{p}\right) \eta+\frac{(1-s)}{l}\left(\frac{d p}{p}\right) \eta}{m \frac{d p}{p}+(1-m) \frac{d Y}{Y}-m \lambda \frac{d r}{r}}$, que pode ser simplificado para $\varepsilon_{M}=\frac{\eta}{l} \frac{\left(\frac{d p}{p}\right)}{m \frac{d p}{p}+(1-m) \frac{d Y}{Y}-m \lambda \frac{d r}{r}}$. 
No denominador, substituir (4a) $\frac{d Y}{Y}=(1-l) \frac{d P}{P}+l\left(\frac{d w}{w}+\frac{d N}{N}\right)$,

que com os mesmos supostos adotados anteriormente se torna

$$
\begin{aligned}
& \frac{d Y}{Y}=(1-l) \frac{d P}{P}+l \frac{d N}{N}, \text { onde } \frac{d N}{N}=\frac{s}{l}\left(\frac{d p}{p}\right) \eta+\frac{(1-s)}{l}\left(\frac{d p}{p}\right) \eta=\frac{\eta}{l}\left(\frac{d p}{p}\right) \text { ou seja, } \\
& \frac{d Y}{Y}=(1-l) \frac{d P}{P}+l\left[\frac{\eta}{l}\left(\frac{d p}{p}\right)\right]=(1-l) \frac{d P}{P}+\eta\left(\frac{d p}{p}\right) .
\end{aligned}
$$

Supondo que os preços variam na mesma proporção que os lucros: $\frac{d Y}{Y}=(1-l+\eta)\left(\frac{d p}{p}\right)$. Ainda no denominador, podemos substituir $\frac{d r}{r}=\pi \frac{d P}{P}-\frac{d p_{I}}{p_{I}}$ que, com o mesmo suposto de que os preços variam na mesma proporção que os lucros, se torna $\frac{d r}{r}=(\pi-1) \frac{d p}{p}$.

Portanto:

$$
\varepsilon_{M}=\frac{\frac{s}{l}\left(\frac{d p_{I}}{p_{I}}-\frac{d w_{I}}{w_{I}}\right) \eta+\frac{(1-s)}{l}\left(\frac{d p_{C}}{p_{C}}-\frac{d w_{C}}{w_{C}}\right) \eta}{m \frac{d p_{I}}{p_{I}}+(1-m) \frac{d Y}{Y}-m \lambda \frac{d r}{r}}=\frac{\eta}{l} \frac{\left(\frac{d p}{p}\right)}{m \frac{d p}{p}+(1-m)(1-l+\eta)\left(\frac{d p}{p}\right)-m \lambda(\pi-1) \frac{d p}{p}} .
$$

Simplificando

$$
\begin{aligned}
& \varepsilon_{M}=\frac{\eta}{l} \frac{1}{m+(1-m)(1-l+\eta)-m \lambda(\pi-1)}=\frac{\eta}{l} \frac{1}{m+1-l+\eta-m+m l-m \eta-m \lambda \pi+m \lambda} . \\
& \varepsilon_{M}=\frac{\eta}{l} \frac{1}{1-l+\eta+m l-m \eta-m \lambda \pi+m \lambda}=\frac{\eta}{l} \frac{1}{1+(\eta-l)(1-m)+m \lambda-m \lambda \pi}:
\end{aligned}
$$


Multiplicando o numerador e o denominador por $1-l$ :

$\varepsilon_{M}=\eta \times \frac{1-l}{l} \times \frac{1}{(1-l)[1+(\eta-l)(1-m)+m \lambda-m \lambda \pi]}$

$\varepsilon_{M}=\eta \times \frac{1-l}{l} \times \frac{1}{(1-l)+\eta-\eta m-l+l m+m \lambda-m \lambda \pi-l \eta+l \eta m+l l-l l m-l m \lambda+l m \lambda \pi}$

$\varepsilon_{M}=\eta \times \frac{1-l}{l} \times \frac{1}{(1-l)+(1-m)(\eta-l-l \eta+l l)+(1-\pi)(m \lambda-\operatorname{lm} \lambda)}$

$\varepsilon_{M}=\eta \times \frac{1-l}{l} \times \frac{1}{(1-l)+(1-m)(1-l)(\eta-l)+(1-\pi) m \lambda(1-l)}$

$\varepsilon_{M}=\eta \times \frac{1-l}{l} \times \frac{1}{(1-l)[1+(1-m)(\eta-l)+(1-\pi) m \lambda]}$

$\varepsilon_{M}=\eta \times \frac{1-l}{l} \times \frac{1}{(1-l)[1+(1-m)(\eta-l)+m \lambda-m \lambda \pi]}$

Embora nosso resultado corrija o resultado de Meade (para quem

$\left.\varepsilon_{M}=\eta \times \frac{1-l}{l} \times \frac{1}{(1-l)(1+\eta[1-m]+m \lambda)-m \lambda \pi}\right)$, isso não altera as conclusões anteriores. 